\title{
Breakage of a thoracic epidural catheter during its removal -A case report-
}

\author{
Yong-Ho Lee, Hee Youn Hwang, Woo-Seok Sim, Mikyung Yang, and Chul Joong Lee \\ Department of Anesthesiology and Pain Medicine, Samsung Medical Center, Sungkyunkwan University School of Medicine, Seoul, \\ Korea
}

The breakage of an epidural catheter is a rare complication during the removal of a thoracic epidural catheter. There are many causes to breakage of an epidural catheter, such as the characteristics of the catheter itself, patient's factors (anatomy, position during insertion and removal of the catheter, and the BMI), and the difficulty of the procedure. Surgical removal is considered if there is a possibility that the retained catheter might cause neurological problems. We experienced a breakage of an epidural catheter during its removal, which led to surgical intervention. To prevent the catheter breakage during its removal, the catheter should be withdrawn without excessive tension. In addition, an understanding of the patient's anatomy is essential. (Korean J Anesthesiol 2010; 58: 569-572)

Key Words: Breakage, Catheter, Complications, Epidural, Thoracic.

Continuous epidural analgesia is used for postoperative pain control, labor analgesia, chronic pain, cancer pain control as well as for anesthesia. Thoracic epidural analgesia is particularly effective for postoperative pain control in thoracic and upper gastrointestinal operations. It also has the advantages of preserving the diaphragm function, early extubation, decrease in atelectasis and pneumonia, early ambulation, and the prevention of intestinal ischemia by improving the microvascular perfusion, which may play a role in lowering the perioperative morbidity and mortality $[1,2]$. However, a range of complications including breakage, migration, kinking, and knotting can occur as the catheter is inserted in the epidural space [3-8]. Although an epidural catheter is normally removed without complications, the catheter can break under a range of conditions, such as the impact on the catheter during insertion or removal. To our knowledge, there are no reports of the breakage of a thoracic epidural catheter except for some on the breakage of a lumbar epidural catheter during its removal. We report the surgical extraction of an epidural catheter that had been cut during a prior attempt to remove it by hand along with a review of the relevant literature regarding how to manage and remove a broken epidural catheter.

\section{Case Report}

A 70-year-old man (155 cm and $52.7 \mathrm{~kg}$ ) presented with progressive dysphagia underwent an endoscopic examination and was diagnosed with esophageal cancer. He did not have a

Received: November 10, 2009. Revised: 1st, November 26, 2009; 2nd, November 26, 2009. Accepted: December 10, 2009.

Corresponding author: Hee Youn Hwang, M.D., [Present address] Department of Anesthesiology and Pain Medicine, Eulji University Hospital, 1306, Dunsan-dong, Seo-gu, Daejeon 301-726, Korea. Tel: 82-42-611-3654, Fax: 82-42-482-9036, E-mail: loveh2y@naver.com

(c) This is an open-access article distributed under the terms of the Creative Commons Attribution Non-Commercial License (http:// creativecommons.org/licenses/by-nc/3.0/), which permits unrestricted non-commercial use, distribution, and reproduction in any medium, provided the original work is properly cited. 
prior medical history of diabetes or hypertension but he had undergone esophageal stent insertion one month earlier due to an esophageal stricture caused by lye ingestion.

Preoperative thoracic epidural catheter insertion was performed for patient controlled analgesia during the postoperative period. Informed consent for the epidural catheter was obtained from the patient after explaining the insertion, objective, and complications. With the patient in the prone position, a pillow was inserted under the chest to maintain the thoracic kyphosis, such as lateral decubitus or sitting position. $10 \%$ povidone-iodine (PVP-I, Betadine) and $75 \%$ alcohol were used to sterilize the area of interest. The left 10 thoracic pedicle was identified for skin insertion using a C-arm and 1\% lidocaine was infiltrated subcutaneously. A 17 G Tuohy needle was inserted via the paramedian approach at the T7-T8 vertebral interlaminar space. Although there was some difficulty in inserting the catheter into the epidural space, after several attempts, the epidural space was confirmed using the loss of resistance technique and a lateral view of the C-arm using contrast. A $19 \mathrm{G}$ epidural catheter (FlexTip Plus ${ }^{\circledR}$, Arrow, USA) was inserted and advanced $3 \mathrm{~cm}$ into the epidural space, i.e. $10 \mathrm{~cm}$ from skin, and the tip of catheter was then located at the upper end plate of the T7 vertebra and fixed with a catheter fixator (Statlock ${ }^{\circledR}$, Bard, USA). There was no significant resistance in the insertion of the epidural catheter and there was no cerebrospinal fluid or blood in the aspiration. An epidural test dose of $3 \mathrm{ml}$ of $1.5 \%$ lidocaine with $1: 200,000$ epinephrine indicated a decrease in sensation between the T4-T8 sensory dermatome in the sensory test using alcohol and there were no significant changes in his vital signs and power of the upper and lower extremities.

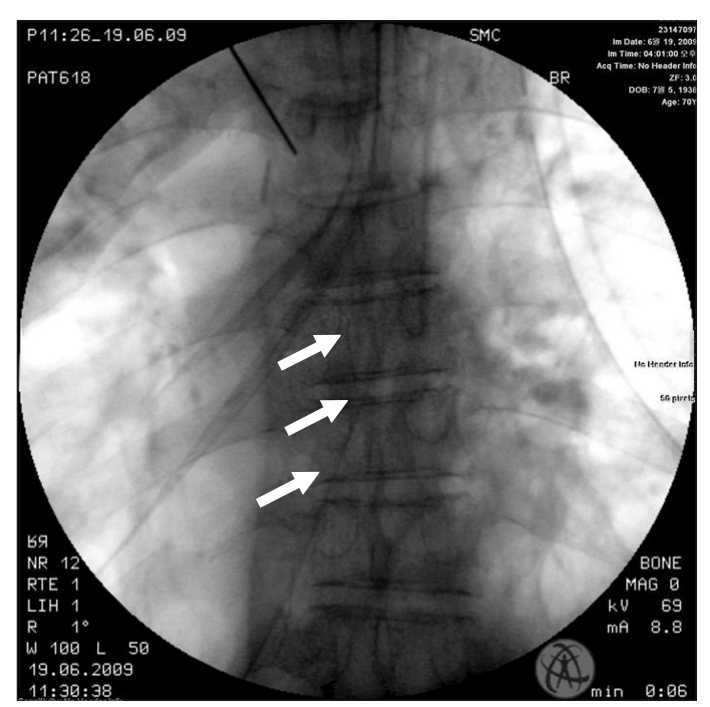

Fig. 1. Thoracic spine anteroposterior X-ray shows the retained epidural catheter (arrows).
The patient underwent an Ivor-Lewis operation according to the schedule and pain control with less than 2 on the visual analogue scale. Three days after surgery, thoracic surgeon attempted to remove the epidural catheter. The patient was placed in the sitting position but removal was difficult. During the removal procedure, the patient did not complain of pain. The thoracic surgeon attempted to remove the catheter by grasping the proximal portion of the catheter in contact with the skin using Kelly. At that time, the epidural catheter broke and the coil remained in the body. The remaining catheter was confirmed to be in the epidural space from a thoracic x-ray (Fig. 1). Because the epidural catheter could be a cause of infection in the epidural space, foreign body removal was scheduled under general anesthesia. The site of the remnant epidural catheter was confirmed using $\mathrm{C}$-arm and the skin was cut from the site of catheter insertion to $7 \mathrm{~cm}$ cephalad. The surgeons gently removed the epidural catheter after exposing the interlaminar space between $\mathrm{T} 7$ and $\mathrm{T} 8$ by dissecting through the layers of subcutaneous tissue and paraspinal muscles. After surgery, the patient was discharged without significant neurological complications.

\section{Discussion}

The breakage of epidural catheters during their removal is rare. In cases where an epidural catheter is broken during its removal, the characteristics of the catheter itself should be considered as one of the causes. The $19 \mathrm{G}$ epidural catheter (FlexTip Plus ${ }^{\circledR}$, Arrow, USA) with a stainless steel coil used in this case has advantages over an nonreinforced catheter, such as easy insertion and infrequent incidence of associated paresthesia and epidural venous cannulation [7]. On the other hand, this type of reinforced catheter was reported to fracture uniformly at $5-6 \mathrm{~cm}$ from the distal tip, where there is less inner metal coil. This fracture of a reinforced catheter occurs at an average tensile strength $<2.24 \mathrm{~kg}$ at $22^{\circ} \mathrm{C}$ (room temperature) and $<1.98 \mathrm{~kg}$ at $37^{\circ} \mathrm{C}$ (body temperature), which is much lower than that at which an nonreinforced catheter breaks $[7,9]$. Therefore, in the case of extensive tensile strength due to some specific reasons, the catheter can break at 5-6 cm from the distal tip. The part inserted in the body is longer than $5-6 \mathrm{~cm}$ so the fractured catheter tip can remain in the body. The length of the catheter in the epidural space may another factor for breakage. If the catheter is long, the epidural catheter can become kinked, bent or knotted, and may be located in the intervertebral foramen. Therefore, it is recommended that the length of the catheter in the epidural space be less than $5 \mathrm{~cm}$ [10]. The factors influencing the catheter removal include the location of insertion, the simplicity of the procedure, and the duration of insertion, etc. [11]. The decrease in durability due to 
catheter damage by the needle edge or cutter during insertion can cause problems during its removal [12].

The spinous process of the thoracic vertebra presents more acute angulation than that of the lumbar vertebra, so the approach of the needle to place an epidural catheter is not easy. Therefore, the needle is usually inserted at two to three vertebral heights below the target epidural space. This means that the relatively longer catheter placed in the body than a lumbar catheter carries a risk of occasional hold in the interlaminar space because the thoracic interlarminar space is narrower than that of the lumbar vertebra [13].

Research aimed at preventing breakage of a lumbar epidural catheter suggests that gentle and continuous tension for removal be applied to the catheter, and removal should be attempted several hours later in cases where the catheter has been stretched [14]. The injection of normal saline and an expansion of the catheter is one of many removal methods [15]. The tensile strength can be reduced when the patient is placed in the same position as they were at the time of insertion [16]. For easy removal, the lateral decubitus position rather than the sitting position is recommended because gravity can be excluded [17]. In cases of difficulty in removing an epidural catheter, the attachment of tape to the patient body with a pull of the catheter can allow removal of the catheter when the patient moves and the obstruction is released [18]. Excessive flexion or extension of the patient's waist is one method. Although there is a report of a catheter being removed using a Tuohy needle over the catheter [19], that method is not recommended because the catheter can be damaged $[7,11]$. Moreover, an ex vivo study suggested that a decrease in tensile strength can increase the likelihood of breakage during removal using the instrument [20].

The earlier mentioned methods for removal would be helpful, even though this case occurred during the removal of a thoracic epidural catheter instead of a lumbar epidural catheter. However, although there is no report of the patient's position during the removal of a thoracic epidural catheter, the patient's position during the removal of a lumbar catheter will be different from that during the removal of a thoracic epidural catheter. A lumbar epidural catheter is normally inserted using the median approach in the lateral decubitus position. The angle between the Tuohy needle and epidural space or skin is more vertical than a thoracic epidural catheter. In addition, there are differences in the structures for insertion between the lumbar and thoracic vertebra.

In cases of difficulty in removing the catheter, before applying excessive tension, which may cause stretching or breakage, it is important to confirm the location of the catheter and evaluate the etiology using imaging tools including computed tomography (CT) [11]. It is not recommended to use a stainless steel hemostat or similar instruments to remove the catheter [20].

If the epidural catheter breaks during removal, the surgical removal should be considered first. The best practice is to assure the patient that neurological complications are quite rare and inform the patient of treatments available in such cases. It is reasonable to remove the epidural catheter surgically in children in order to reduce the possibility of neurological problems through growth and development or in adults with definite neurological symptoms as well as in cases in whom the catheter tip is located in the dura [11].

In this case, the difficulty in the procedure due to the anatomical difficulty, i.e. the acute angle between the epidural catheter and patient's back, might place a relatively long length in the body, which may cause excessive tensile strength during its removal. The breakage of catheter was also attributed to the use of Kelly. A change in the patient's position or a time interval might prevent catheter breakage. While there are papers on the tensile strength for removing a lumbar epidural catheter, there are no reports on the tensile strength during the removal of a thoracic epidural catheter. Therefore, more study will be needed to measure the tensile strength during the removal of a thoracic epidural catheter.

\section{References}

1. Clemente A, Carli F. The physiological effects of thoracic epidural anesthesia and analgesia on the cardiovascular, respiratory and gastrointestinal systems. Minerva Anestesiol 2008; 74: 549-63.

2. Ulke ZS, Senturk M. Non-analgesic effects of thoracic epidural anesthesia. Agri 2007; 19: 6-12.

3. Kim JI, Kim DK, Lee JW, Son JS. The Breakage of Epidural Catheter during continuous Epidural Analgesia for Cancer Pain -A case report-. Korean J Anesthesiol 2007; 53: 513-5.

4. Hobaika AB. Breakage of epidural catheters: etiology, prevention, and management. Rev Bras Anestesiol 2008; 58: 227-33.

5. Bishton IM, Martin PH, Vernon JM, Liu WH. Factors influencing epidural catheter migration. Anaesthesia 1992; 47: 610-2.

6. Roddin MJ, Dancey FM. Kinking of epidural catheters. Anaesthesia 2000; 55: 831.

7. Asai T, Yamamoto K, Hirose T, Taguchi H, Shingu K. Breakage of epidural catheters: a comparison of an arrow reinforced catheter and other nonreinforced catheters. Anesth Analg 2001; 92: 246-8.

8. Karraz MA. Knotting of an epidural catheter like a tie. Anesth Analg 2002; 95: 257.

9. Tsui BC, Finucane B. Tensile strength of 19- and 20-gauge arrow epidural catheters. Anesth Analg 2003; 97: 1524-6.

10. Browne RA, Politi VL. Knotting of an epidural catheter: a case report. Can Anaesth Soc J 1979; 26: 142-4.

11. Mitra R, Fleischmann K. Management of the sheared epidural catheter: is surgical extraction really necessary? J Clin Anesth 2007; 19: 310-4.

12. Collier C. Epidural catheter breakage: a possible mechanism. Int J 
Obstet Anesth 2000; 9: 87-93.

13. Murata H, Sakai T, Goto S, Sumikawa K. Three-dimensional computed tomography for difficult thoracic epidural needle placement. Anesth Analg 2008; 106: 654-8.

14. Pierre HL, Block BM, Wu CL. Difficult removal of a wire-reinforced epidural catheter. J Clin Anesth 2003; 15: 140-1.

15. Gadalla F. Removal of a tenacious epidural catheter. Anesth Analg 1992; 75: 1071-2.

16. Morris GN, Warren BB, Hanson EW, Mazzeo FJ, DiBenedetto DJ. Influence of patient position on withdrawal forces during removal of lumbar extradural catheters. Br J Anaesth 1996; 77: 419-20.
17. Blackshear RH, Gravenstein N, Radson E. Tension applied to lumbar epidural catheters during removal is much greater with patient sitting versus lying. Anesthesiology 1991; 75 : A833.

18. Woehlck HJ, Bolla B. Uncoiling of wire in arrow flextip epidural catheter on removal. Anesthesiology 2000; 92: 907-9.

19. Shantha TR, Mani M. A simple method to retrieve irretrievable epidural catheters. Anesth Analg 1991; 73: 508-9.

20. Nishio I, Sekiguchi M, Aoyama Y, Asano S, Ono A. Decreased tensile strength of an epidural catheter during its removal by grasping with a hemostat. Anesth Analg 2001; 93: 210-2. 